\title{
Mean-field study of the interplay between antiferromagnetism and d-wave superconductivity
}

\author{
Bumsoo Kyung \\ Département de physique and Centre de recherche sur les propriétés électroniques de matériaux avancés. \\ Université de Sherbrooke, Sherbrooke, Québec, Canada J1K 2R1
}

(April 17, 2000)

\begin{abstract}
The interplay between antiferromagnetism and d-wave superconductivity is studied in a meanfield approximation for a generic microscopic Hamiltonian with short-range repulsion and nearneighbor attraction. In the presence of competing microscopic interactions, the phase boundaries of antiferromagnetic and superconducting states are significantly modified in some region of the doping-temperature plane. The transition between superconductivity and antiferromagnetism occurs through a phase where both order parameters coexist with a third, dynamically generated, spin-triplet amplitude. This dynamical generation of a new order parameter is not restricted to a system with antiferromagnetism and d-wave superconductivity, but is a generic feature for fermionic systems. The dynamically generated spin-triplet order parameter is found to be robust to variations in the mean-field Hamiltonian.
\end{abstract}

PACS numbers: 71.10.Fd, 71.27.+a

The close interplay between antiferromagnetism (AF) and superconductivity (SC) has been of great interest both to experimentalists and theoretical physicists for many years. The competition of two different order parameters has been observed in various systems such as organic superconductors, [1] ternary compounds, [2] heavy fermion compounds [3] as well as high temperature superconductors [4] in the latest. For some compounds, such as the cuprates, the AF and SC phases are close to each other but separated, while for other compounds such as organic superconductors, ternary compounds and heavy fermion compounds the two phases often touch and sometimes even coexist in the phase diagram. This competition problem of two different orders was extensively studied in a mean-field approximation first for s-wave superconductors [5] and later for anisotropic superconductors [6,7]. Recently the same problem including the fluctuation effect of AF and SC was considered by Onoda and Imada [8,9] in the framework of self-consistent renormalization theory.

A few years ago Zhang and his coworkers 10,11] proposed that the SC and AF phases, particularly, of the high $T_{c}$ cuprates might be related to each other in a unified theory based on $\mathrm{SO}(5)$ symmetry. In this $\mathrm{SO}(5)$ theory, the SC components of the five dimensional superspin vector are transformed to the $\mathrm{AF}$ components by, so called, $\pi$ operators. These operators carry charge 2 and spin 1 , and total momentum $Q=(\pi, \pi)$ in two dimensions. Further the above authors showed that a sharp resonance of $41 \mathrm{meV}$ observed in inelastic neutron scattering experiments [12] might be a direct consequence of the existence of $\pi$ operators. To the author's knowledge, the possibility of spin-triplet amplitude with total momentum $Q$ often called $\pi$-triplet in the literature, was first found by Psaltakis and Fenton [13] in their mean-field study of the competition problem of AF and SC. These authors showed that when a spin-density wave (SDW) coexists with spin-singlet Cooper-pair superconductivity among the same electrons, a non-zero spin-triplet amplitude must appear. Recently a similar problem was studied by Murakami and Fukuyama [14.15] by invoking the g-ology diagrams in one-dimensional electron system. But in the above two works the authors did not study the condition of appearance of the spin-triplet amplitude and its robustness. Furthermore in previous mean-field studies the manner how the AF-only and SC-only phase boundaries are modified into new phase boundaries in the presence of two competing interactions, was not well investigated. Thus the purpose of this paper is twofold: First, detailed phase diagrams are presented in the doping $(x=1-n)$-temperature $(T)$ plane in the presence of competing interactions and second the condition of appearance of the spin-triplet order parameter and its robustness are clarified.

In this paper we study a phenomenological mean-field Hamiltonian where antiferromagnetism and d-wave superconductivity are built in on equal footing.

$$
\begin{aligned}
H & =\sum_{\vec{k}, \sigma}\left(\xi_{\vec{k}}-\mu\right) c_{\vec{k}, \sigma}^{+} c_{\vec{k}, \sigma}+U \sum_{i}\left(c_{i, \uparrow}^{+} c_{i, \uparrow}\left\langle c_{i, \downarrow}^{+} c_{i, \downarrow}\right\rangle+\text { H.C. }\right) \\
& -V \sum_{i}\left(\Delta_{d, i}^{+}\left\langle\Delta_{d, i}\right\rangle+\text { H.C. }\right)-W \sum_{i}\left(\Delta_{t, i}^{+}\left\langle\Delta_{t, i}\right\rangle+\text { H.C. }\right)
\end{aligned}
$$

A possible spin-triplet pair amplitude $\left\langle\Delta_{t, i}\right\rangle$ is also considered in general for self-consistency and its significance will become clear later. In particular, we will see that it is dynamically generated, even when $W=0$. The sin- 
glet $\Delta_{d, i}$ and triplet $\Delta_{t, i}$ pair destruction operators are defined as

$$
\begin{aligned}
\Delta_{d, i} & =\frac{1}{2} \sum_{\delta} g(\delta)\left(c_{i+\delta, \uparrow} c_{i, \downarrow}-c_{i+\delta, \downarrow} c_{i, \uparrow}\right) \\
\Delta_{t, i} & =\frac{1}{2} \sum_{\delta} g(\delta)\left(c_{i+\delta, \uparrow} c_{i, \downarrow}+c_{i+\delta, \downarrow} c_{i, \uparrow}\right),
\end{aligned}
$$

and the structure factor $g(\delta)$ is chosen to have a d-wavelike form such as

$$
g(\delta)= \begin{cases}1 / 2 & \text { if } \delta=( \pm 1,0) \\ -1 / 2 & \text { if } \delta=(0, \pm 1) \\ 0 & \text { if otherwise }\end{cases}
$$

Later it will be also clear why the same structure factor $g(\delta)$ is introduced in the spin-triplet term. The strength of AF, SC, and spin-triplet pair interactions is governed by $U, V$, and $W$, respectively, which are all positive in our study. Essentially the same form of the meanfield Hamiltonian was previously studied by Psaltakis and Fenton, [13] and Kato and Machida, [6] and $\mathrm{Mu}-$ rakami and Fukuyama 14] except for some slight differences. $\xi_{\vec{k}}$ is a tight binding energy dispersion given as $\xi_{\vec{k}}=-2 t\left(\cos k_{x}+\cos k_{y}\right)-4 t^{\prime} \cos k_{x} \cos k_{y}$, where $t$ and $t^{\prime}$ are hopping constants for nearest neighbors and next nearest neighbors. And $\mu$ is the chemical potential which controls the particle density $n$.

In a mean-field approximation, three different order parameters $m, s$, and $t$ corresponding to the three different interactions may be defined in the following way:

$$
\begin{aligned}
& \left\langle c_{i, \sigma}^{+} c_{i, \sigma}\right\rangle=\left\langle n_{i, \sigma}\right\rangle=\frac{n}{2}+\sigma m \cos \left(\vec{Q} \cdot \vec{R}_{i}\right) \\
& \frac{1}{2} \sum_{\delta} g(\delta)\left\langle c_{i+\delta, \uparrow} c_{i, \downarrow}-c_{i+\delta, \downarrow} c_{i, \uparrow}\right\rangle=s \\
& \frac{1}{2} \sum_{\delta} g(\delta)\left\langle c_{i+\delta, \uparrow} c_{i, \downarrow}+c_{i+\delta, \downarrow} c_{i, \uparrow}\right\rangle=t \cos \left(\vec{Q} \cdot \vec{R}_{i}\right),
\end{aligned}
$$

where $\vec{Q}$ is the (commensurate) antiferromagnetic wave vector equal to $(\pi, \pi)$ in two dimensions. Now the meanfield Hamiltonian $H_{M F}$ is quadratic in the original electron operator and in terms of a new four component field operator $\psi_{\vec{k}}$ it becomes bilinear

$$
H_{M F}=\sum_{\vec{k}}^{\prime} \psi_{\vec{k}}^{+} M_{\vec{k}} \psi_{\vec{k}}+E_{0},
$$

where

$$
\psi_{\vec{k}}^{+}=\left(c_{\vec{k}, \uparrow}^{+}, c_{-\vec{k}, \downarrow}, c_{\vec{k}+\vec{Q}, \uparrow}^{+}, c_{-\vec{k}-\vec{Q}, \downarrow}\right) .
$$

The matrix $M_{\vec{k}}$ is given as

$$
M_{\vec{k}}=\left(\begin{array}{cccc}
\varepsilon_{\vec{k}} & V s \phi(\vec{k}) & -U m & W t \phi(\vec{k}) \\
V s \phi(\vec{k}) & -\varepsilon_{\vec{k}} & -W t \phi(\vec{k}) & -U m \\
-U m & -W t \phi(\vec{k}) & \varepsilon_{\vec{k}+\vec{Q}} & -V s \phi(\vec{k}) \\
W t \phi(\vec{k}) & -U m & -V s \phi(\vec{k}) & -\varepsilon_{\vec{k}+\vec{Q}}
\end{array}\right)
$$

where

$$
\varepsilon_{\vec{k}}=\xi_{\vec{k}}-\mu
$$

and $\phi(\vec{k})=\cos k_{x}-\cos k_{y}$ is the Fourier transform of $g(\delta)$. The prime symbol on the summation requires the summation of wave vectors in half of the first Brillouin zone, in order to take into account the doubling of a magnetic unit cell in the presence of (commensurate) AF order. The constant energy shift $E_{0}$ depending on $m, s$, $t$, and $\mu$ is given as

$$
E_{o}=N\left(U m^{2}+V s^{2}+W t^{2}-\mu\right),
$$

where $N$ is the total number of lattice sites. The energy eigenvalues of $M_{\vec{k}}$ yield four energy dispersions $\pm E_{ \pm}(\vec{k})$,

$$
\begin{aligned}
E_{ \pm}(\vec{k})= & {\left[\left(\varepsilon_{\vec{k}}^{2}+\varepsilon_{\vec{k}+\vec{Q}}^{2}\right) / 2+(U m)^{2}+(V s \phi(\vec{k}))^{2}\right.} \\
& \left.+(W t \phi(\vec{k}))^{2} \pm g(\vec{k})\right]^{1 / 2},
\end{aligned}
$$

where $g(\vec{k})$ is given as

$$
\begin{aligned}
g(\vec{k})= & {\left[\left(\varepsilon_{\vec{k}}^{2}-\varepsilon_{\vec{k}+\vec{Q}}^{2}\right)^{2} / 4+\left(\varepsilon_{\vec{k}}-\varepsilon_{\vec{k}+\vec{Q}}\right)^{2}(W t \phi(\vec{k}))^{2}\right.} \\
& \left.+\left(\left(\varepsilon_{\vec{k}}+\varepsilon_{\vec{k}+\vec{Q}}\right)(U m)+2(V s \phi(\vec{k}))(W t \phi(\vec{k}))\right)^{2}\right]^{1 / 2} .
\end{aligned}
$$

When $s=t=0$ or $m=t=0$, the energy eigenvalue reduces to that of SDW or BCS approximation of the corresponding interactions.

The free energy is easily obtained either from the trace formula or from the Feynman theorem

$$
F=-2 T \sum_{\vec{k}}^{\prime} \sum_{\alpha= \pm} \log \left(2 \cosh \frac{E_{\alpha}(\vec{k})}{2 T}\right)+E_{o} .
$$

Now the three mean-field equations are obtained by the stationary condition of $F$ with respect to the corresponding order parameters $\frac{\partial F}{\partial m}=\frac{\partial F}{\partial s}=\frac{\partial F}{\partial t}=0$ and one more unknown constant $\mu$ is determined by the thermodynamic relation $n=-\frac{\partial F}{\partial \mu}$. The resulting four equations are

$$
\begin{aligned}
m & =\frac{1}{2 N} \sum_{\vec{k}}^{\prime} \sum_{\alpha= \pm}\left\{(U m)+\alpha \frac{\left(\varepsilon_{\vec{k}}+\varepsilon_{\vec{k}+\vec{Q}}\right)}{2 g(\vec{k})}\left[\left(\varepsilon_{\vec{k}}+\varepsilon_{\vec{k}+\vec{Q}}\right)(U m)\right.\right. \\
& +2(V s \phi(\vec{k}))(W t \phi(\vec{k}))]\} \frac{1}{E_{\alpha}}(\vec{k}) \tanh \left(\frac{\beta E_{\alpha}(\vec{k})}{2}\right) \\
s & =\frac{1}{2 N} \sum_{\vec{k}}^{\prime} \sum_{\alpha= \pm} \phi(\vec{k})\left\{(V s \phi(\vec{k}))+\alpha \frac{(W t \phi(\vec{k}))}{g(\vec{k})}\left[\left(\varepsilon_{\vec{k}}+\varepsilon_{\vec{k}+\vec{Q}}\right)\right.\right. \\
& (U m)+2(V s \phi(\vec{k}))(W t \phi(\vec{k}))]\} \frac{1}{E_{\alpha}}(\vec{k}) \tanh \left(\frac{\beta E_{\alpha}(\vec{k})}{2}\right)
\end{aligned}
$$




$$
t=\frac{1}{2 N} \sum_{\vec{k}}^{\prime} \sum_{\alpha= \pm} \phi(\vec{k})\left\{(W t \phi(\vec{k}))+\alpha \frac{(W t \phi(\vec{k}))\left(\varepsilon_{\vec{k}}-\varepsilon_{\vec{k}+\vec{Q}}\right)^{2}}{2 g(\vec{k})} \quad \begin{array}{l}
\text { dependent of } t, \\
(V s \phi(\vec{k}))\left(\varepsilon_{\vec{k}}+\varepsilon_{\vec{k}+\vec{Q}}\right)(U m) .
\end{array}\right.
$$

$$
\begin{aligned}
& +\alpha \frac{(V s \phi(\vec{k}))}{g(\vec{k})}\left[\left(\varepsilon_{\vec{k}}+\varepsilon_{\vec{k}+\vec{Q}}\right)(U m)\right. \\
& +2(V s \phi(\vec{k}))(W t \phi(\vec{k}))]\} \frac{1}{E_{\alpha}}(\vec{k}) \tanh \left(\frac{\beta E_{\alpha}(\vec{k})}{2}\right)
\end{aligned}
$$$$
n=1-\frac{1}{2 N} \sum_{\vec{k}}^{\prime} \sum_{\alpha= \pm}\left\{\left(\varepsilon_{\vec{k}}+\varepsilon_{\vec{k}+\vec{Q}}\right)\right.
$$$$
+\alpha \frac{\left(\varepsilon_{\vec{k}}+\varepsilon_{\vec{k}+\vec{Q}}\right)\left(\varepsilon_{\vec{k}}-\varepsilon_{\vec{k}+\vec{Q}}\right)^{2}}{2 g(\vec{k})}
$$$$
+\alpha \frac{2(U m)}{g(\vec{k})}\left[\left(\varepsilon_{\vec{k}}+\varepsilon_{\vec{k}+\vec{Q}}\right)(U m)\right.
$$$$
+2(V s \phi(\vec{k}))(W t \phi(\vec{k}))]\} \frac{1}{E_{\alpha}}(\vec{k}) \tanh \left(\frac{\beta E_{\alpha}(\vec{k})}{2}\right) .
$$

Although essentially the same form of the mean-field Hamiltonian was studied by Psaltakis and Fenton, 13 and Murakami and Fukuyama 14], the final mean-field equations are somewhat different. In Ref. [13] the Fermi surface is divided into two regions. In region one the nesting condition $\varepsilon_{\vec{k}}=-\varepsilon_{\vec{k}+\vec{Q}}$ is satisfied and in the other region it is not satisfied. Thus the mean-field equations are different in different regions. Even after taking this into account there are still some discrepancies. In Ref. [14] apparently there are several erroneous terms in the mean-field equations. As a double check for the correctness of the above mean-field equations, we also computed the transformation matrix relating the original $c$ operator to the eigen-operator $\gamma$ and with it obtained the same mean-field equations. All the components of the matrix are given in the Appendix.

Before plunging into numerical calculations, we point out several distinctive features inferred from the structure of the mean-field equations itself. First, a given order parameter is affected by the other ones in a more direct manner than for the case where only two order parameters, $\mathrm{AF}$ and $\mathrm{SC}$, [6] are considered. For the latter case the influence from the other order parameters comes indirectly through $E_{\alpha}(\vec{k})$ and $g(\vec{k})$. Second, in spite of non-vanishing driving potentials for the three different order parameters, only a state with a single order parameter or with three order parameters together is possible. For the former case the proof is obvious. Suppose two of the three order parameters, for example, $m$ and $s$, are non-vanishing and then examine whether the third one $(t)$ can vanish or not. Since the equations have the same structure with respect to the three order parameters, this particular choice suffices for general purpose. The right hand of the $t$ equation, Eq. 15, has a term which is in-
Thus unless this term is exactly canceled by the combination of the other terms, the third order parameter $t$ does not vanish. But perfect cancellation is impossible because the term Eq. 17 cannot be written as a linear combination of the other terms in Eq. 15. Third, despite

(15) the vanishing driving potential for one of the order parameters, for example, $W=0$, the corresponding order parameter $t$ (in this case) can be dynamically generated. Since the terms containing $t$ enter with the combination of $W t \phi(\vec{k})$ in the right hand of Eq. 15, only Eq.17 survives for $W=0$. As long as both $\mathrm{AF}$ and SC order parameters have non-vanishing amplitude, namely, they coexist, $t$ also has non-vanishing amplitude even with $W=0$.

This last feature was first discussed by Psaltakis and Fenton [13]. They argued that when AF and SC order parameters $\left\langle c_{\vec{k}, \uparrow}^{+} c_{\vec{k}+\vec{Q}, \uparrow}\right\rangle,\left\langle c_{\vec{k}, \uparrow} c_{-\vec{k}, \downarrow}\right\rangle$ have non-vanishing value at the same time, two electrons $c_{\vec{k}+\vec{Q}, \uparrow}, c_{-\vec{k}, \downarrow}$ are coupled to the same electron $c_{\vec{k}, \uparrow}^{+}$through the Bogoliubov transformation, thus they are not generally independent but instead may form another order parameter $\left\langle c_{\vec{k}+\vec{Q}, \uparrow} c_{-\vec{k}, \downarrow}\right\rangle$. Eq.17 shows in which circumstances this may happen. The d-wave factor $\phi(\vec{k})$ in front of the curly bracket in Eq. 15 comes from the spin-triplet term and $\phi(\vec{k})$ in Eq.17 from the spin-singlet term, thus the same symmetry factors, or at least those which are not orthogonal to each other, are required to obtain a non-vanishing third order parameter. At half-filling for a nearest neighbor model $\left(t^{\prime}=0\right)$ where $\varepsilon_{\vec{k}}+\varepsilon_{\vec{k}+\vec{Q}}=0$, however, the dynamical generation of another order parameter does not happen. Since the equations again have the same structure with respect to the three order parameters, $m$ $(s)$ can be dynamically generated with $U=0(V=0)$ as well. In this paper we argue that this dynamical generation of third order parameter in the coexistence state of two orders is not restricted only to the case with $\mathrm{AF}$ and d-wave SC orders, but is a generic feature for fermionic systems. With a proper choice of the symmetry factor, a third order parameter can be dynamically generated without the corresponding driving potential. For example, for the case of SDW and p-wave SC, the third order parameter is $\sum_{\vec{k}} \phi(\vec{k})\left\langle c_{\vec{k}, \uparrow} c_{\vec{k}+\vec{Q}, \downarrow}\right\rangle$, where $\phi(\vec{k})$ is a p-wave form factor such as $\sin k_{x}$ or $\sin k_{y}$. This may be called $\pi$-singlet in parallel to the $\pi$-triplet for the case of SDW and d-wave SC. In SO(5) theory, the $\pi$-triplet amplitude is not an order parameter. It is the generator of infinitesimal rotations between $\mathrm{AF}$ and $\mathrm{SC}$ order parameters. In the mixed $\mathrm{AF}+\mathrm{SC}+\pi$-triplet phase that is discussed here, the $\pi$-triplet itself acquires a non-zero expectation value.

First we start with presenting phase diagrams of the nearest neighbor model $\left(t^{\prime}=0\right)$. In this paper, $t$, lattice spacing, $\hbar$, and $k_{B}$ are set to be unity, and $U$ is fixed at 
half of the bandwidth, 4 . With varying $V$, the shape of the phase diagram changes significantly. In the present study we restrict ourselves to a particular case where at half filling the ground state is an antiferromagnetic insulator, which might be more relevant especially for high $T_{c}$ cuprates. This requirement dictates that $V$ is less than $U=4$ and thus we consider two different values of $V, 3$ and 1.5. In Fig. 1 and in the following figures, the dotted and dashed curves denote the phase boundary of $\mathrm{AF}$ and $\mathrm{SC}$ without the driving interaction term for the other order parameter. For instance, the dotted curve in Fig. If (a) is the phase boundary of AF state with $V=0$ and the dashed one of SC state with $U=0$. Fig.1 clearly shows how the AF-only (dotted curve) and SConly (dashed curve) phase boundaries change into new ones (solid curves) in the presence of two competing interactions. For $V=1.5$ the major change of the phase boundary appears in the low doping region of the $\mathrm{SC}$ state. Near half-filling, due to strong local repulsion, the SDW state is formed throughout the lattice and it strongly breaks time-reversal symmetry, leading to the destruction of the SC state. But the AF-only phase remains virtually unchanged at this strength. In the low doping region a new phase appears where the $\mathrm{AF}$ and SC and $\pi$-triplet order parameters are non-vanishing, as described earlier. For $V=3.0$ where the strengths of $\mathrm{AF}$ and SC interactions become comparable, both the AFonly and SC-only phase boundaries significantly change. As in $V=1.5$ the SC phase boundary is most strongly modified near half-filling, while the AF phase boundary far away from half-filling where AF order is most vulnerable to other interactions. Because of the larger value of $V$, the SC phase region becomes wider than that of $V=1.5$. At a particular point where four different phases (paramagnetic metal, $\mathrm{SC}, \mathrm{AF}$, and $\mathrm{AF}+\mathrm{SC}+\pi$-triplet phases) meet, a tetracritical point [16,11] appears. That tetracritical point does not have $\mathrm{SO}(5)$ symmetry in general, but it has $\mathrm{SO}(3) \otimes \mathrm{U}(1)$ symmetry. $\mathrm{SO}(5)$ symmetry is realized only when $t^{\prime}=0$. 117

In Fig. 2 we consider phase diagrams with the next nearest neighbor hopping $\left(t^{\prime}=-0.3\right)$. This value is close to what is suggested for $\mathrm{YBa}_{2} \mathrm{Cu}_{3} \mathrm{O}_{7-\delta}$ and $\mathrm{Bi}_{2} \mathrm{Sr}_{2} \mathrm{CaCu}_{2} \mathrm{O}_{8+\delta}$. For small $V$ the coexistence region is more suppressed near half-filling than that for $t^{\prime}=0$ in Fig.1. (a) because the effective density of states for SC is reduced. For $V=3$, however, the coexistence region is more strongly modified far away from half-filling than in the $t^{\prime}=0$ case, since the increased effective strength for $\mathrm{SC}$ in this doping range pushes the $\mathrm{AF}$ phase to the left. The slight increase of the AF region near $x=0.3$ with $t^{\prime}=-0.3$ is presumably due to an artifact of the commensurate SDW approximation.

As in the previous figures, the dotted and dashed curves in Fig. 3 denote the order parameters of AF with $V=0$ and those of SC with $U=0$, respectively. The order parameters are plotted in Fig. 3 -(a) as a function of doping $(x)$ with temperature fixed at 0.3 . As can be inferred from Fig.1. (b), the order parameters of AF and SC states are most strongly affected far away from half-filling and near half-filling, respectively, by the presence of competing interactions. Where two order parameters (AF, $\mathrm{SC}$ ) coexist, the third $\pi$-triplet order parameter appears without its driving potential $(W=0)$. Its amplitude is maximum when those of $\mathrm{AF}$ and $\mathrm{SC}$ become comparable as is implied in Eq.17 and the maximum value is approximately one fifth of those of AF and SC at the corresponding doping level. When one of the order parameters appears or disappears, the other order parameter changes in its slope, a characteristic feature of a meanfield approximation. In Fig. 35(b) the order parameters are plotted as a function of temperature at fixed doping 0.1. Because of their competing nature, both order parameters are reduced at low temperatures from those of AF-only and SC-only phases.

In Fig. 1 the phase diagram is shown for $W=3, V=3$, and $t^{\prime}=0$. As in the previous figures, the dotted, dashed, and long-dashed curves are phase boundaries for AF with $V=W=0$, for SC with $U=W=0$ and for $\pi$-triplet with $U=V=0$. Due to the $W$ term, $\mathrm{AF}$ and $\mathrm{SC}$ regions are increased compared with those for $W=0$ case (Fig.1 (b)). This is because the $W$ term increases the stability of $\mathrm{AF}$ and $\mathrm{SC}$ by converting $\mathrm{SC}(\mathrm{AF})$ electrons into $\mathrm{AF}$ (SC) electrons instead of destroying them. One of the interesting features is that a $\pi$-triplet phase does not appear as a separate phase for $W=3$, although $W$ is as large as $V$. In order to create such a phase, $W \geq 4.5$ is required. Then a SC-only phase disappears in the phase diagram and the roles of SC and $\pi$-triplet are interchanged. In this situation the SC order parameter appears only where AF and $\pi$-triplet phases coexist.

In the last figure the order parameters are plotted as a function of doping and temperature for $W=3$, $V=3, t^{\prime}=0$. As before the dotted, dashed, and long-dashed curves are the order parameters for AF with $V=W=0$, for SC with $U=W=0$ and for $\pi$-triplet with $U=V=0$, respectively. Because of the $W$ term, the critical concentration of AF order at $T=0.3$ (Fig.5 (a)) is increased from $x=0.2$ for $W=0$ to $x=0.27$, much closer to that of AF order with $V=W=0$ (dotted curve). Thus the order parameter of AF with $W=3$, is similar to that of AF with $V=W=0$. The maximum value of $\pi$-triplet order parameter is $0.051,0.064$, and 0.091 for $W=0,1.5$, and 3.0, respectively. This indicates the robustness of dynamically generated $(W=0)$ part of the $\pi$-triplet order parameter.

Before closing several comments are in order. In order to find the nature of phase transition at the phase boundary of $\mathrm{AF}$ (or $\mathrm{SC}) \leftrightarrow \mathrm{AF}+\mathrm{SC}+\pi$-triplet, we calculated the free energies of the three different phases (AF, SC, and $\mathrm{AF}+\mathrm{SC}+\pi$-triplet) at constant temperature and chemical potential. Inside the phase boundary the free energy of $\mathrm{AF}+\mathrm{SC}+\pi$-triplet is lowest [18] and 
its slope does not change as the temperature crosses the phase boundary. Thus in a mean-field approximation the phase transition of $\mathrm{AF}$ (or SC) $\leftrightarrow \mathrm{AF}+\mathrm{SC}+\pi$-triplet is second order. [19] This is consistent with the earlier result of Kato and Machida. [7] These authors classified anisotropic pairing states enumerated group theoretically into two categories: less-competing and more-competing states according to the combined symmetry of the SC order parameter (parity and translational symmetry determined by the nesting vector). Less-competing states yield second order phase transition between AF (or SC) and $\leftrightarrow \mathrm{AF}+\mathrm{SC}+\pi$-triplet, while more-competing states yield first order phase transition. D-wave SC belongs to the less-competing states according to their classification. In this paper only a commensurate magnetic structure is considered in the SDW approximation. From more sophisticate considerations, 20] away from half-filling and at low temperatures the commensurate magnetic order should be replaced by an incommensurate one. Lastly, mean-field theories neglect fluctuations completely, thus they are inadequate in low dimensions because there is no long-range order at finite temperatures in one and two dimensions. 21] This pathological feature may be avoided by considering a small z-axis component. Since strong fluctuations still remain due to quasi two dimensional nature, the phase boundaries will be shrunk drastically. For AF state the boundary will be squeezed toward the low temperature and low doping, while for SC state toward the low temperature. Accordingly the coexistence region may remain in a very small region of the phase diagram or even disappear. 22 Then the mean-field critical temperatures will be replaced by a crossover temperature $T^{*}$ below which a pseudogap [23] will appear in the single particle spectral function. This precursor effect of the ground state can be caused by antiferromagnetic or superconducting fluctuations depending on the location in the phase diagram. Likewise, a strongly fluctuating $\pi$-triplet amplitude will manifest itself in the region surrounded by the AF and SC mean-field phase boundaries.

In conclusion, we have studied the close interplay between antiferromagnetism and d-wave superconductivity in a mean-field approximation. In the presence of competing interactions the phase boundaries of AF and $\mathrm{SC}$ states are significantly modified in some region of the doping-temperature plane. In the coexistence region of $\mathrm{AF}$ and d-wave SC a new order of spin-triplet pair is dynamically generated without its driving interaction. We argue that this feature is not restricted only to the current problem, but is a generic feature for fermionic systems. The dynamical generation of spin-triplet order parameter is found to be robust to variations in the meanfield Hamiltonian. Fluctuation effects to the mean-field result are briefly discussed.

The author would like to thank Prof. Tremblay for numerous help in various stages of this work as well as for critical reading of the paper. He also thanks Prof.
Sénéchal for helpful discussions in the early stage. The present work was supported by a grant from the Natural Sciences and Engineering Research Council (NSERC) of Canada and the Fonds pour la formation de Chercheurs et d'Aide à la Recherche (FCAR) of the Québec government.

\section{APPENDIX: TRANSFORMATION MATRIX}

The transformation matrix $A_{i, j}$ relating the original $c$ operator to the eigen-operator $\gamma$ is defined by

$$
\psi_{i}(\vec{k})=\sum_{j} A_{i, j} \gamma_{j}(\vec{k}) .
$$

The components of the first column vector are given as

$$
\begin{aligned}
A_{1,1} & =\left(\left(E_{+}(\vec{k})+\varepsilon_{\vec{k}}\right)\left(E_{+}(\vec{k})+\varepsilon_{\vec{k}+\vec{Q}}\right)\left(E_{+}(\vec{k})-\varepsilon_{\vec{k}+\vec{Q}}\right)\right. \\
& -\left(E_{+}(\vec{k})-\varepsilon_{\vec{k}+\vec{Q}}\right)(U m)^{2}-\left(E_{+}(\vec{k})+\varepsilon_{\vec{k}}\right)(V s \phi(\vec{k}))^{2} \\
& -\left(E_{+}(\vec{k})+\varepsilon_{\vec{k}+\vec{Q}}\right)(W t \phi(\vec{k}))^{2}+2(U m)(V S \phi(\vec{k})) \\
& (W t \phi(\vec{k}))) / \sqrt{\sum_{i} A_{i, 1}^{2}} \\
A_{2,1}= & \left(( V S \phi ( \vec { k } ) ) \left[\left(E_{+}(\vec{k})+\varepsilon_{\vec{k}+\vec{Q}}\right)\left(E_{+}(\vec{k})-\varepsilon_{\vec{k}+\vec{Q}}\right)\right.\right. \\
& \left.-(U m)^{2}-(V S \phi(\vec{k}))^{2}+(W t \phi(\vec{k}))^{2}\right] \\
& \left.+2(U m)(W t \phi(\vec{k})) \varepsilon_{\vec{k}+\vec{Q}}\right) / \sqrt{\sum_{i} A_{i, 1}^{2}} \\
A_{3,1}= & -\left(( U m ) \left[\left(E_{+}(\vec{k})+\varepsilon_{\vec{k}}\right)\left(E_{+}(\vec{k})+\varepsilon_{\vec{k}+\vec{Q}}\right)\right.\right. \\
& \left.-(U m)^{2}-(V S \phi(\vec{k}))^{2}-(W t \phi(\vec{k}))^{2}\right] \\
+ & \left.(V s \phi(\vec{k}))(W t \phi(\vec{k}))\left(2 E_{+}(\vec{k})+\varepsilon_{\vec{k}+\vec{Q}}+\varepsilon_{\vec{k}}\right)\right) \\
& / \sqrt{\sum_{i} A_{i, 1}^{2}} \\
& \left.+(U m)(V s \phi(\vec{k}))\left(\varepsilon_{\vec{k}+\vec{Q}}+\varepsilon_{\vec{k}}\right)\right) / \sqrt{\sum_{i} A_{i, 1}^{2}}, \\
A_{4,1}= & \left(( W t \phi ( \vec { k } ) ) \left[\left(E_{+}(\vec{k})+\varepsilon_{\vec{k}}\right)\left(E_{+}(\vec{k})-\varepsilon_{\vec{k}+\vec{Q}}\right)\right.\right. \\
- & \left.(U m)^{2}+(V S \phi(\vec{k}))^{2}-(W t \phi(\vec{k}))^{2}\right] \\
+ & \left.(U)^{2}\right)
\end{aligned}
$$

where the square of normalization of the vector is

$$
\begin{aligned}
& \sum_{i} A_{i, 1}^{2}=2 E_{+}(\vec{k}) g(\vec{k})\left[\left(E_{+}(\vec{k})+\varepsilon_{\vec{k}}\right)\right. \\
& \left(2 g(\vec{k})+\varepsilon_{\vec{k}}^{2}-\varepsilon_{\vec{k}+\vec{Q}}^{2}\right)+2(U m)^{2}\left(\varepsilon_{\vec{k}}+\varepsilon_{\vec{k}+\vec{Q}}\right) \\
& \left.+4(U m)(V S \phi(\vec{k}))(W t \phi(\vec{k}))+2(W t \phi(\vec{k}))^{2}\left(\varepsilon_{\vec{k}}-\varepsilon_{\vec{k}+\vec{Q}}\right)\right] .
\end{aligned}
$$

By symmetry the second column vector $A_{i, 2}$ is obtained from the first one as follows: $A_{1,2}=-A_{2,1}, A_{2,2}=$ 
$A_{1,1}, A_{3,2}=A_{4,1}, A_{4,2}=-A_{3,1}$. The third column vector may be expressed in terms of the first one: $A_{1,3}=$ $A_{3,1}, A_{2,3}=-A_{4,1}, A_{3,3}=A_{1,1}, A_{4,3}=-A_{2,1}$ with the following replacement in $A_{i, 1}, \varepsilon_{\vec{k}} \leftrightarrow \varepsilon_{\vec{k}+\vec{Q}}, g(\vec{k}) \leftrightarrow$ $-g(\vec{k}), E_{+}(\vec{k}) \leftrightarrow E_{-}(\vec{k})$. Finally the fourth column vector $A_{i, 4}$ is found from the third one by symmetry $A_{1,4}=A_{2,3}, A_{2,4}=-A_{1,3}, A_{3,4}=-A_{4,3}, A_{4,4}=A_{3,3}$.

[1] D. Jerome and H. J. Schulz, Adv. Phys. 31, 299 (1982); D. Jerome, in Proceedings of the IV Conference on Superconductivity in d-and f-band Metals (Karlsruhe), edited by W. Buckel and W. Weber, 421 (1982).

[2] Superconductivity in Ternary Compounds, edited by M. B. Maple and ØFisher (Springer-Verlag, New York, 1982), Vols. 1 and 2.; R. N. Shelton, L. S. HausermannBerg, M. J. Johnson, P. Klavins, and H. D. Yang, Phys. Rev. B 34, 199 (1986).

[3] C. Broholm, J. K. Kjems, G. Aeppli, Z. Fisk, J. L. Smith, S. M. Shapiro, G. Shirane, and H. R. Ott, Phys. Rev. Lett. 58, 917 (1987); D. E. Cox, G. Shirane, S. M. Shapiro, G. Aeppli, Z. Fisk, J. L. Smith, J. K. Kjems, and H. R. Ott, Phys. Rev. B 33, 3614 (1986); B. Batlogg, D. J. Bishop, E. Bucher, B. Golding Jr., A. P. Ramirez, Z. Fisk, J. L. Smith, and H. R. Ott, J. Magn. Magn. Mater. 63-64, 441 (1987).

[4] J. G. Bednorz and K. A. M'uller, Z. Phys. B 64, 189 (1986); C. Almasan and M. B. Maple in Chemistry of High Temperature Superconductors, edited by C. N. R. Rao (World Scientific, Singapore, 1991).

[5] K. Machida, J. Phys. Soc. Jpn. 50, 2195 (1981); K. Machida and T. Matsubara, ibid, 50, 3231 (1981); K. Machida , ibid, 51, 1420 (1982).

[6] M. Kato and K. Machida, J. Phys. Soc. Jpn. 56, 2136 (1987).

[7] M. Kato and K. Machida, Phys. Rev. B 37, 1510 (1988).

[8] S. Onoda and M. Imada, J. Phys. Soc. Jpn. in press and cond-mat/9903030.

[9] S. Onoda and M. Imada, cond-mat/9908195.

[10] E. Demler and S. C. Zhang, Phys. Rev. Lett. 75, 4126 (1995).

[11] S. C. Zhang, Science, 275, 1089 (1997).

[12] H. A. Mook, M. Yethiraj, G. Aeppli, T. E. Mason, and T. Armstrong, Phys. Rev. Lett. 70, 3490 (1993).

[13] G. C. Psaltakis and E. W. Fenton, J. Phys. C 16, 3913 (1983).

[14] M. Murakami and H. Fukuyama, J. Phys. Soc. Jpn. 67, 2784 (1998).

[15] M. Murakami, cond-mat/9904213.

[16] M. E. Fisher and D. R. Nelson, Phys. Rev. Lett. 32, 1350 (1974).

[17] C. L. Henley, Phys. Rev. Lett. 80, 3590 (1998).

[18] Recently several studies have shown that similar meanfield Hamiltonians have microscopic inhomogeneous stripe solutions. See J. Zaanen and O. Gunnarsson, Phys.
Rev. B 40, 7391 (1989), and I. Martin, G. Ortiz, A. V. Balatsky, and A. R. Bishop, cond-mat/0003316.

[19] Note that the $\mathrm{AF}+\mathrm{SC}+\pi$-triplet phase transforms as a subgroup of either the $\mathrm{AF}$ or the $\mathrm{SC}$ phases, as required from general considerations.

[20] H. J. Schulz, Phys. Rev. Lett. 64, 1445 (1990).

[21] N. D. Mermin and H. Wagner, Phys. Rev. Lett. 17, 1133 (1966).

[22] Another mechanism that could lead to the disappearance of the $\mathrm{AF}+\mathrm{SC}+\pi$-triplet phase is disorder. Indeed, the triplet component of the mixed phase should be very sensitive to disorder.

[23] Y. Vilk and A. M. Tremblay, J. Phys. I (France) 7, 1309 (1997). 

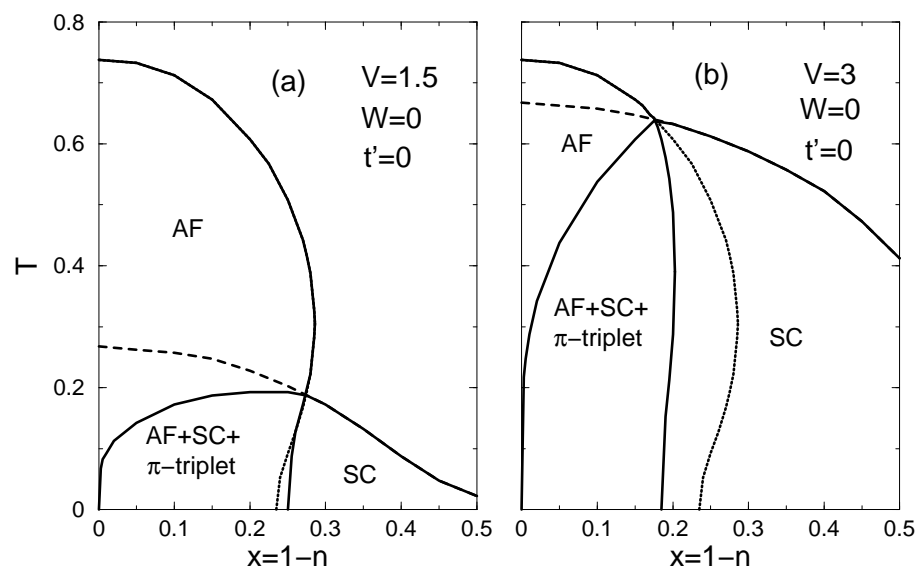

FIG. 1. Phase diagrams in doping $(x=1-n)$ and temperature ( $T$ ) plane for (a) $V=1.5$, and (b) $V=3$, with $U=4$, $W=0$, and $t^{\prime}=0$. The dotted and dashed curves denote the phase boundaries of AF with $V=0$ and of SC with $U=0$, respectively.
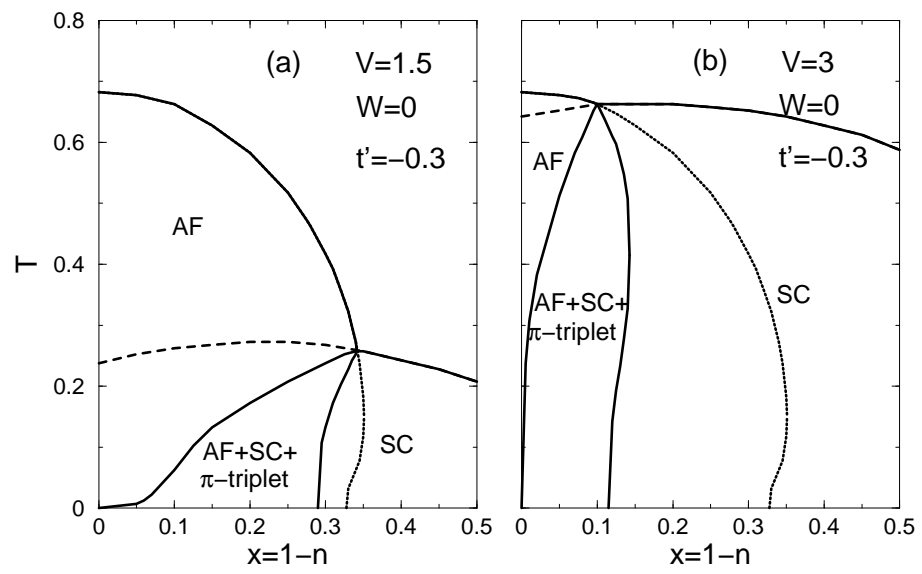

FIG. 2. Phase diagrams in doping $(x=1-n)$ and temperature (T) plane for (a) $V=1.5$, and (b) $V=3$, with $U=4$, $W=0$, and $t^{\prime}=-0.3$. The dotted and dashed curves denote the phase boundaries of AF with $V=0$ and of SC with $U=0$, respectively.
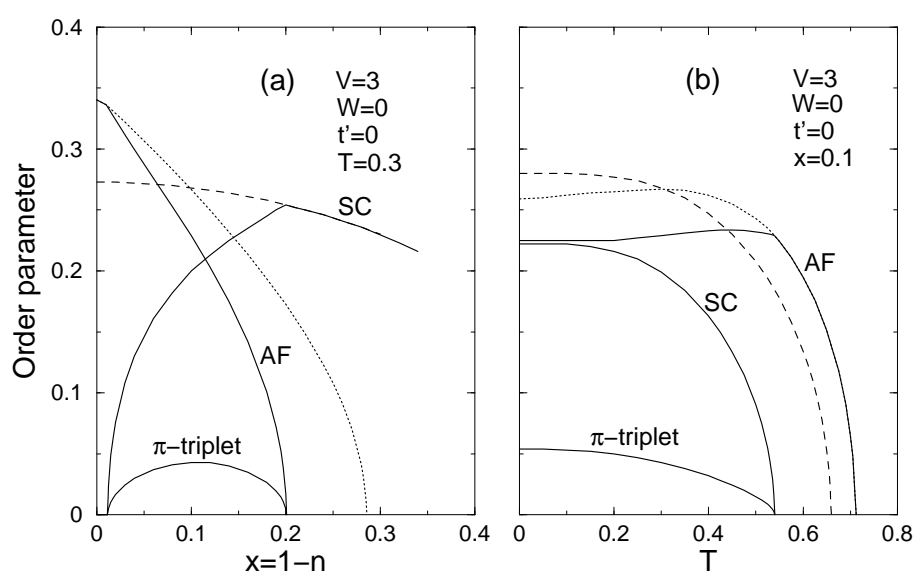

FIG. 3. Order parameters at (a) $T=0.3$ and (b) $x=0.1$, for $U=4, V=3, W=0$, and $t^{\prime}=0$. The dotted and dashed curves denote the order parameters of AF with $V=0$ and of SC with $U=0$, respectively.

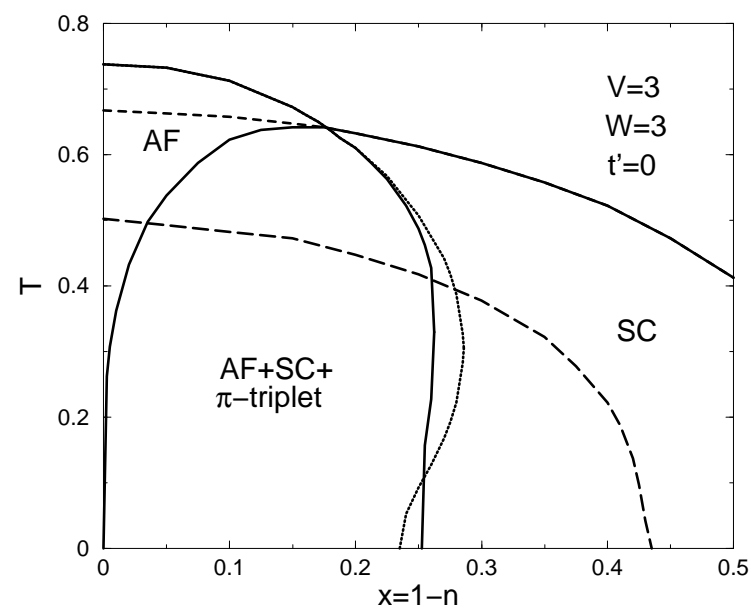

FIG. 4. Phase diagram in doping $(x=1-n)$ and temperature $(T)$ plane for $U=4, V=3, W=3$, and $t^{\prime}=0$. The dotted, dashed, and long-dashed curves denote the phase boundaries of AF with $V=W=0$, of SC with $U=W=0$, of $\pi$-triplet with $U=V=0$, respectively. 

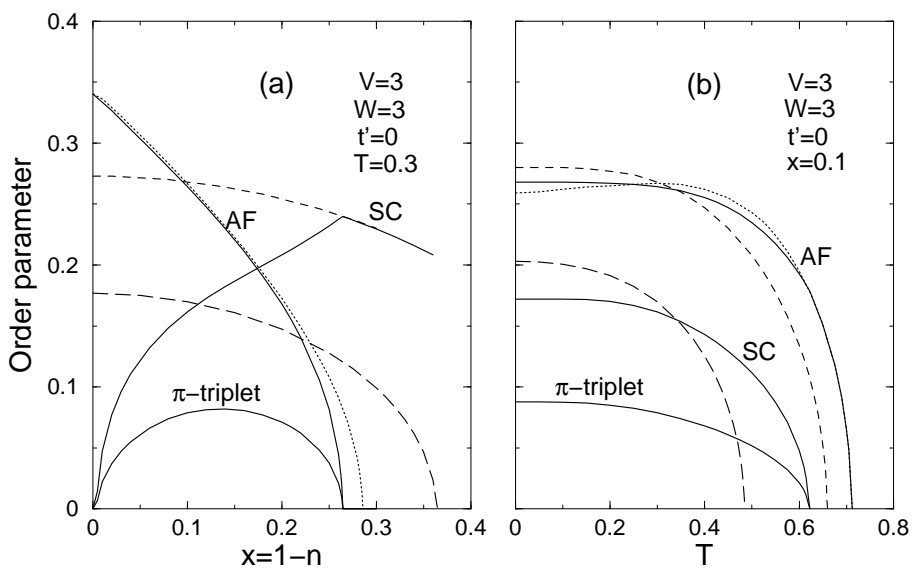

FIG. 5. Order parameters at (a) $T=0.3$ and (b) $x=0.1$, for $U=4, V=3, W=3$, and $t^{\prime}=0$. The dotted, dashed, and long-dashed curves denote the order parameters of $\mathrm{AF}$ with $V=W=0$, of SC with $U=W=0$, of $\pi$-triplet with $U=V=0$, respectively. 